\title{
Observation of Grease Fluidity in a Ball Bearing Using Neutron Imaging Technology
}

\author{
Kazumi Sakai $^{1)^{*}}$, Yusuke Ayame ${ }^{1)}$, Yoshimu Iwanami ${ }^{2)}$, Nobuharu Kimura $^{2)}$ and Yoshihiro Matsumoto ${ }^{3)}$ \\ ${ }^{1)}$ Grease R\&D Group, Lubricants R\&D Dept., ENEOS Corporation, \\ 8 Chidoricho, Naka-ku, Yokohama, Kanagawa 231-0815, Japan \\ ${ }^{2)}$ Analytical Technology Group, R\&D Solution Center, Central Technical Research Laboratory, ENEOS Corporation, \\ 8 Chidoricho, Naka-ku, Yokohama, Kanagawa 231-0815, Japan \\ ${ }^{3)}$ Neutron Science and Technology Center, Comprehensive Research Organization for Science and Society, \\ 162-1 Shirakata, Tokai, Naka, Ibaraki 319-1106, Japan \\ *Corresponding author: Kazumi Sakai (sakai.kazumi@eneos.com)
}

Manuscript received 30 March 2021; accepted 17 May 2021; published 15 June 2021

\begin{abstract}
The grease fluidity and migration inside a ball bearing has been visualized non-destructively by using a neutron imaging technology. It has been clarified that a lithium (Li) complex grease with lower torque lubricates in the channeling state and that a single Li soap grease with higher torque lubricates in the churning state. Adhesion of the Li complex grease to bearing balls was quite limited, and most of the grease stayed on cage surfaces between the balls; adhesion of the single Li soap grease to bearing balls was remarkable. These observation results correlate to their grease performances for the bearing torque. The less adhesion of the Li complex grease to bearing balls contributes to reducing bearing torque due to easy rotations of bearing balls. In contrast, the remarkable adhesion of the single Li soap grease causes higher shear resistance for bearing ball rotations. The neutron imaging technology clarified the mechanism of the bearing torque difference depending on the grease types and could contribute to developments of energy-saving greases.
\end{abstract}

\section{Keywords}

grease, neutron imaging, bearing, channeling, churning, lubrication

\section{Introduction}

Developments of energy-saving technologies especially in the fields of automotive and machine industries have been progressed over years in order to preserve the global environments and suppress the global warming. It was reported that electric energy consumption of industry motors used for such as pumps, compressors, blowers is estimated to account for about $40 \%$ of the total consumption in Japan [1]; therefore, improvements of the energy efficiency of industry motors are crucial. Rolling element bearings are widely used for axis rotations in motors and lubricated with greases. Greases contribute to prolong bearing service lives; however, there is a conflict that the efficiency of motors declines due to the energy loss (or torque) caused by grease resistance through bearing rotations. Nitta et al. [2] stated that the causes of energy loss in rolling element bearings can be roughly divided into the stirring resistance of lubricants, the viscous rolling resistance, the friction resistance between balls and races, and the friction resistance between balls and cages. Cousseau et al. [3] investigated the influence of greases on thrust ball bearing using a rolling bearing friction torque model. For lowering the energy loss, selecting a low viscosity base oil contributes to reducing the viscous rolling resistance. In addition, grease around bearing races largely influences on the stirring resistance of lubricants (greases), therefore, grease fluidity in bearings plays an essential role in the efficiency of motors.

The grease fluidity can be illustrated by channeling and churning states. In the churning state, a grease is sheared by moving parts such as balls and causes high bearing torque due to drag losses [4,5]. This continuous grease shearing raises temperature of the bearing. The grease shearing leads to grease softening causing lubricant leakage from the bearing. In contrast, in the channeling state, most of grease is pushed away from the running track. The drag losses from grease shearing decrease due to this grease clearing. As a result, the bearing torque reaches steady low values. In the initial phase of a bearing operation, the grease lubricates in the churning state, but a quick transition to the channeling state can occur, which is promising for developing an energy-saving grease.

Visualizations of greases in bearings are required to understand the grease fluidity, however, methods for nondestructive observations of the inside of bearings are limited. Noda et al. [6] investigated grease distributions in bearings 
and captured the transient phenomena between the churning and channeling states by the X-ray computed tomography (CT) imaging. However, some special materials such as fluororesin, fluororesin carbon fiber composite, and glass for cages, rings, and balls of bearings were needed for the observation, because heavy elements such as Fe absorb X-ray strongly. Therefore, it can be stated that there is a hurdle to observe lubricants composed of light elements inside steel bearings using X-ray. Haruyama et al. [7] visualized grease distributions in bearings using a grease with fluorescence particles. In this case, visible light needs to pass through the bearing, therefore, an outer ring comprised of transparent material has to be applied for irradiation of laser light to the grease and observation of the fluorescence from the grease.

In contrast, the neutron imaging does not require such special materials for the non-destructive observation [8]. Compared to $\mathrm{X}$-ray, the interactions of neutron to light elements such as $\mathrm{H}$ and $\mathrm{C}$ are strong but the interactions to heavy elements such as Fe are weak. Therefore, neutron passes through a steel bearing and identifies lubricants inside the bearing. In addition, energy of neutron is low and influences on greases by the irradiation are negligible. These features of neutron are appropriate for non-destructive observations of grease behaviors inside conventional bearings and can contribute to understanding of the grease fluidity. The objective in this study is to visualize the grease distribution inside bearings using the neutron imaging technology. By comparing the greases with different bearing torque behaviors which determines energy-saving performance, the influence of the grease fluidity was discussed.

\section{Experimental methods}

\subsection{Grease samples}

Two types of lithium (Li) greases; Grease A and B were used in this study. As grease thickeners, Li complex and single $\mathrm{Li}$ soap were selected for Grease A and B, respectively. Li complex consists of Li-12-hydroxy stearate and Li-azelate, and single Li soap is Li-stearate. API Group-I base oil with a viscosity grade VG32 was commonly used for both greases. Group-I is mineral oil composed of paraffinic, naphthenic, and aromatic hydrocarbons. The components of each grease and some properties are listed in Table 1.

\subsection{Bearing torque}

In order to evaluate the energy-saving performance of the greases, bearing tests were conducted by using our original bearing friction torque testing machine. For each measurement,

Table 1 Sample greases

\begin{tabular}{|c|c|c|c|c|}
\hline \multicolumn{3}{|l|}{ Grease } & A & B \\
\hline \multirow[t]{2}{*}{ Thickener } & \multicolumn{2}{|l|}{ Li complex, \% } & 12 & - \\
\hline & \multicolumn{2}{|l|}{ Li stearate, $\%$} & - & 14 \\
\hline \multicolumn{3}{|c|}{ Worked penetration (ISO 2137) } & 265 & 270 \\
\hline \multirow[t]{4}{*}{ Base oil } & \multicolumn{2}{|l|}{ Mineral oil (API Group I) } & & \\
\hline & \multirow[t]{2}{*}{ Kinetic viscosity, $\mathrm{mm}^{2} / \mathrm{s}$} & $40^{\circ} \mathrm{C}$ & & \\
\hline & & $100^{\circ} \mathrm{C}$ & & \\
\hline & \multicolumn{2}{|l|}{ Viscosity index } & \multicolumn{2}{|c|}{107} \\
\hline
\end{tabular}

a new 6204 bearing was used. The bearing was washed with white gasoline in order to remove the filled grease before a test grease was applied to the bearing. The bearing was filled with 2 grams of the test grease, which is equivalent to approximately $35 \%$ of the space volume of the bearing. The space volume of the bearing is defined as the maximum volume which a grease can be filled in.

The inner ring of the bearing with the test grease was attached to the main shaft of the tester. After applying radial and thrust loads to the bearing in a housing, a wire, which was connected to a load cell, was attached to the bearing housing in order to detect bearing torque. The bearing assembly was placed in a chamber to control temperature by air convection. After stabilizing temperature at $25^{\circ} \mathrm{C}$ in the chamber, the main shaft was rotated at discrete speeds with stepwise increase (200, 500, 1000, and $2000 \mathrm{~min}^{-1}$ ). The measurement time for 200, 500, and $1000 \mathrm{~min}^{-1}$ was 5 minutes each and for $2000 \mathrm{~min}^{-1}$ was 30 minutes.

\subsection{Neutron imaging}

The bearing with each grease sample was rotated at 2000 $\mathrm{min}^{-1}$ for different durations before the neutron imaging as listed in Table 2. Neutron radiography and computed tomography (CT) measurements of the greases distributed in the bearings were performed by using RADEN [9] in the Materials and Life Science Experimental Facility (MLF) of the Japan Proton Accelerator Research Complex (J-PARC) [10]. RADEN is an apparatus specializing in energy-resolved and conventional neutron imaging using the intense pulsed neutron beam. The bearing sample with grease was fixed on a rotating stage and irradiated by the neutron beam. Neutrons which penetrated the sample were converted to visible light by the ${ }^{6} \mathrm{LiF} / \mathrm{ZnS}$ scintillator screen of $0.10 \mathrm{~mm}$ thickness, and then captured by a water-cooled CCD camera with $2048 \times 2048$ pixels. In the radiography observation neutrons were irradiated in the axial direction of the bearing with a filed-of-view of $80 \mathrm{~mm} \times$ $80 \mathrm{~mm}$ and camera exposure time of about $30 \mathrm{sec}$. The spatial resolution of obtained transmission images was about $60 \mu \mathrm{m}$. As for the CT observation, the 3-dimensional slice images were reconstructed by using the filtered-back-projection method from the 600 transmission images that were obtained by rotating the sample from $0^{\circ}$ to $360^{\circ}$ in 0.6 degree increments.

\section{Results and discussion}

3.1 Bearing torque

The energy-saving performances of the greases were determined by the bearing torque tests. Figure 1 compares the bearing torque of Grease A and B at different rotation speeds. Grease A with Li complex thickener showed lower bearing torque at all rotation speeds compared with Grease B with Li soap thickener. This result may indicate that Grease A lubricates

Table 2 Bearing preparation for neutron imaging

\begin{tabular}{l|l}
\hline Bearing & 6204 with shield \\
\hline Grease content & $2 \mathrm{~g}$ \\
\hline Rotational speed & $2000 \mathrm{~min}^{-1}$ \\
\hline Rotating time & $0,1,10,60 \mathrm{~min}$. \\
\hline Temperature & $25^{\circ} \mathrm{C}$ \\
\hline
\end{tabular}




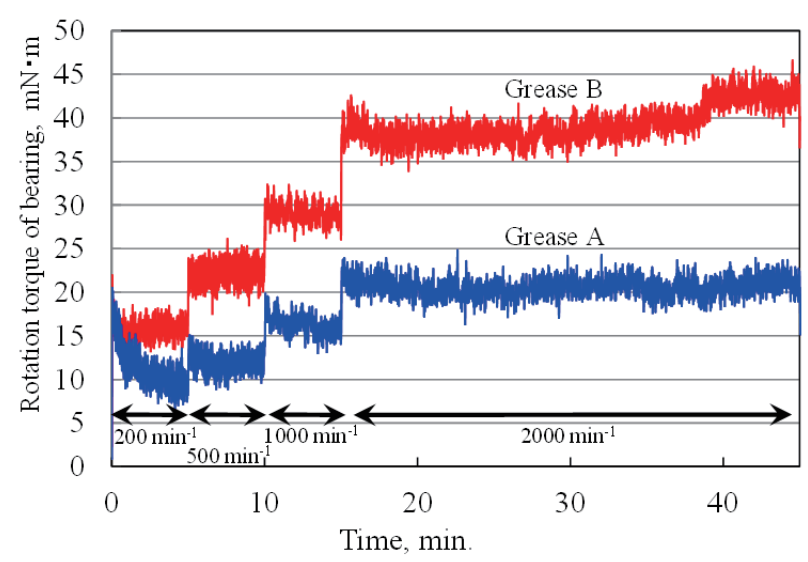

Fig. 1 Bearing torque performance of sample greases

in the channeling state and Grease B lubricates in the churning state under these test conditions. In order to clarify this hypothesis, the neutron imaging was performed as described in the following section.

\subsection{Neutron imaging}

Figure 2 illustrates images of the neutron radiography of the bearings lubricated with the two types of greases after bearing rotations in the axial direction. A photograph of the bearing without a grease taken by a normal camera is also shown in the left of Fig. 2. Image processing for these images was performed so that the gray value becomes brighter as the neutron transmittance decreases in order to make it easier to understand the correspondence with CT results described later. Therefore, light color indicates presence of lubricant with low neutron transmission, and dark color indicates metallic parts with high neutron transmission. Both greases were initially positioned on the cage surfaces before bearing rotations as observed in the figures labeled ' 0 min'. The grease distributions have changed through the bearing rotations from 0 to 60 minutes. Focusing on the grease distribution after 60 minutes rotation, the different grease fluidities were observed depending on the thickener types. The lubricant was observed at the edges of the outer ring for Grease B (as shown in a red circle in Fig. 2), in contrast, most part of Grease A stayed on the cage surfaces. The oil leakage from the bearing shields occurred after 60 minutes bearing rotation for Grease B, not for Grease A, which corresponds to the imaging results.

The images of neutron CT of the bearings in the axial and radial directions are shown in Fig. 3. The observations were conducted on the bearings with the two greases after 60 minutes rotations in order to investigate the grease fluidity in detail. The axial views at the middle plane of the bearings are shown in the left of Fig. 3. Similar to the results of radiography, Grease A was observed mainly on the cage surfaces and Grease B on the cage surfaces areas was less than Grease A. Instead of the cage surfaces, the grease and/or leaked base oil from Grease B were found at the edges of the outer ring as in the radiography image. The adhesion of Grease B on the balls was also observed. The images of the radial direction and the magnified images are shown in the middle and right of Fig. 3, respectively. The adhesion of Grease A to balls is negligible and the voids between the balls and the inner/outer rings are found, which corresponds to the channeling state. It was also found that most of Grease A remained on the cage surfaces, as shown in the radiography image in Fig. 2. On the contrary, the adhesion of Grease B to balls is obvious in the images of the radial direction, which correlates to the churning state. The grease and/or leaked base oil from Grease B were observed inside of the bearing shields, which indicates the oil leakage from bearing shields. Figure 1 shows the bearing torque increase with Grease B at the end of the test. It can be stated that the adhesion of the grease to the balls may increase the viscous resistance between the balls and the cage because the bled oil or grease with a low thickener concentration of Grease B filled the vicinity of the contact.

\subsection{Lubrication mechanism}

Figure 4 illustrates the lubrication mechanisms of Grease

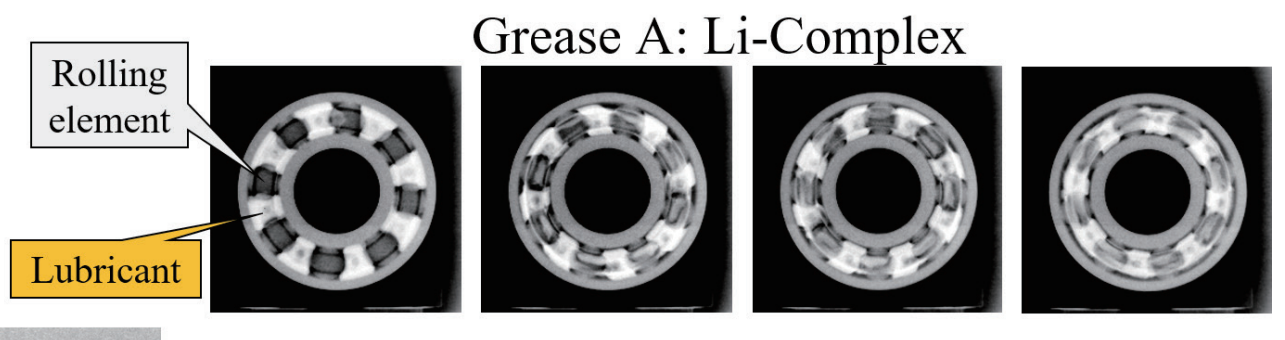

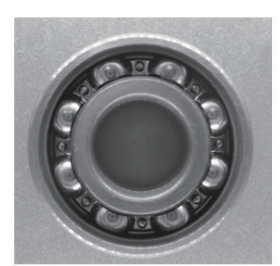

Bearing without grease

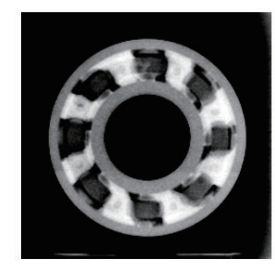

0 min.

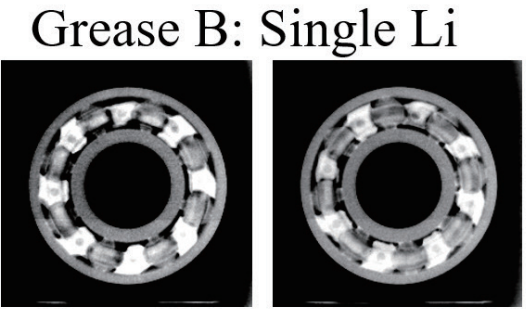

$10 \mathrm{~min}$.

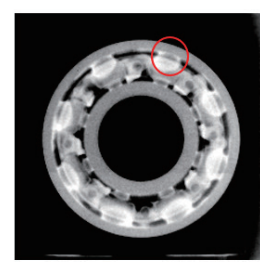

$60 \mathrm{~min}$.

Fig. 2 Neutron radiography of bearings under grease lubrication after rotation for each duration (Red circle: Grease B at the edge of the outer ring) 


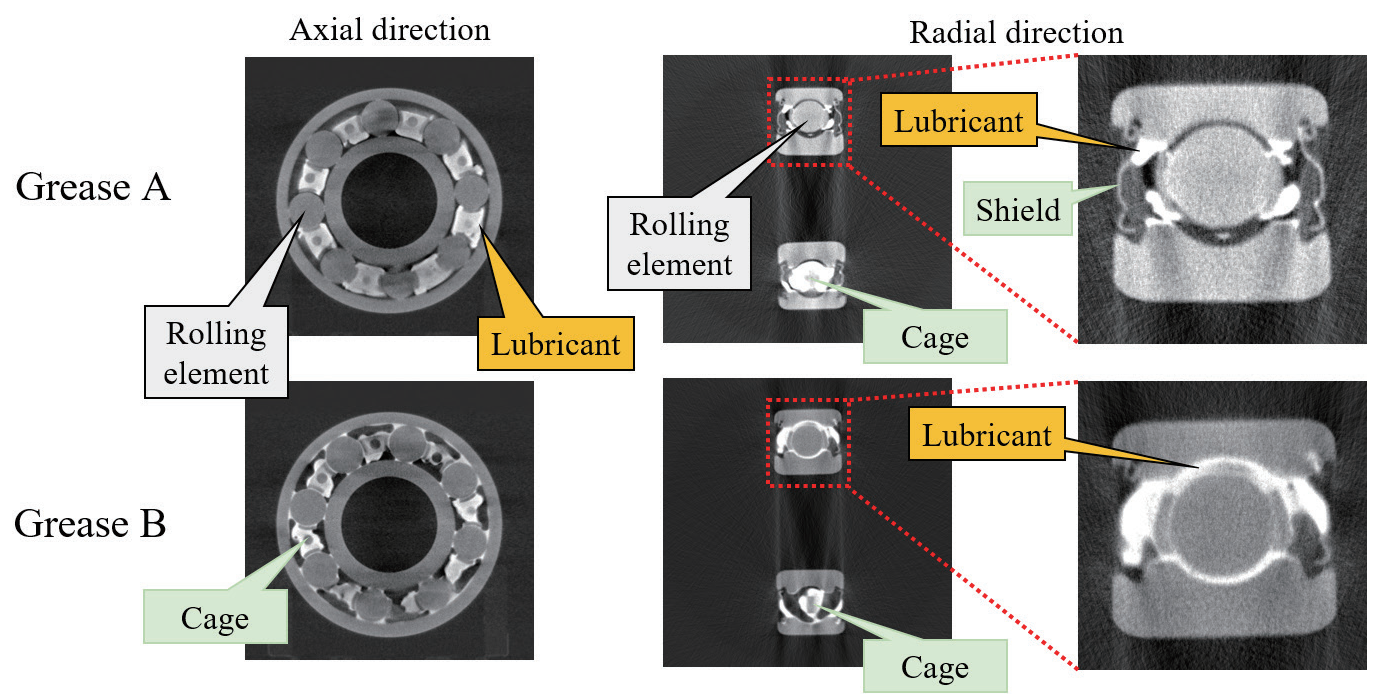

Fig. 3 Neutron computed tomography of bearings under grease lubrication after rotation (60 min.)
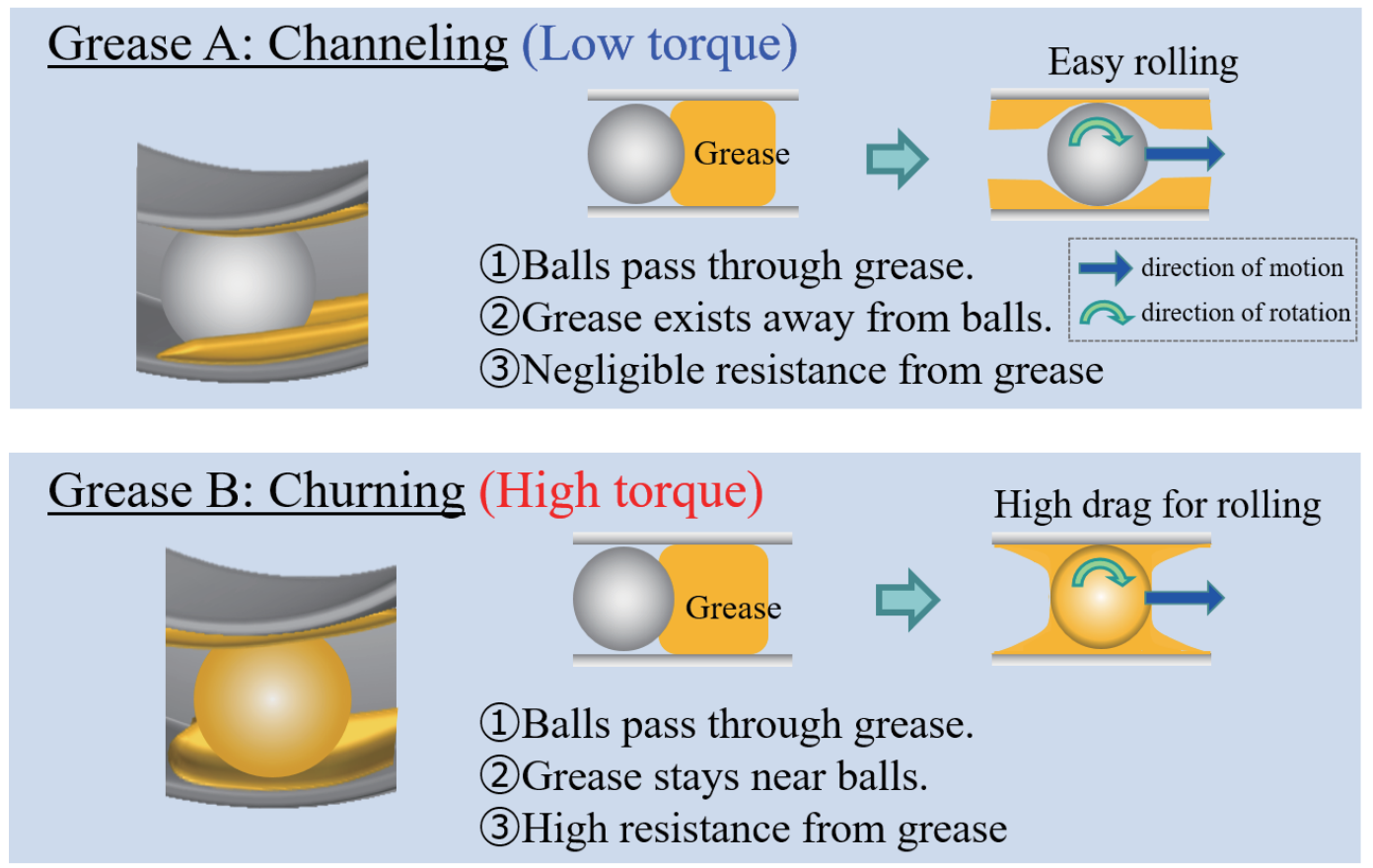

Fig. 4 Lubrication images of two types of greases

A and B from the point of view of channeling and churning states based on the results in this study. Grease A thickened by Li complex lubricates in the channeling state to show lower bearing torque. When the bearing balls pass through Grease A with bearing rotations, most of grease stays on the cage surfaces and hardly stick to the balls, as shown in the images of the neutron CT. A small amount of grease is dragged into contact areas. As a result, the bearing balls rotate smoothly with lower stirring resistance of the grease to decrease bearing torque. In contrast, Grease B thickened by single Li soap lubricates in the churning state to increase bearing torque. The images of neutron CT have revealed that Grease B sticks to bearing balls with bearing rotations, leading to higher drag for ball rolling. The adhesion of the grease to the balls may increase the viscous resistance between the balls and the cage. The grease stuck to balls could be softened by continual shearing, which causes oil and/or grease leakage from the bearing shields. The bled oil or grease with a low thickener concentration is more easily stuck to balls. The neutron imaging technology are very useful to clarify the relations between the grease behaviors inside bearings and the grease formulations, which could contribute to the further developments of energy-saving and long-life greases.

\section{Conclusions}

The neutron imaging technology has visualized grease fluidity and distribution inside ball bearings. The images have captured the crucial grease behaviors such as channeling and churning states which strongly influence on the energy-saving performance. The Li complex thickened grease lubricates in 
channeling state in the bearing with minimal adhesion of the grease to the bearing balls to show lower bearing torque by the smooth ball rotations. Single Li soap grease lubricates in churning state in the bearing with remarkable adhesion of the grease to bearing balls to show higher bearing torque by increasing stirring and viscous resistance. The neutron imaging has a possibility to reveal the grease behaviors inside machinery parts non-destructively, which could contribute to developments of greases with superior performances.

\section{Acknowledgements}

This work was conducted by using RADEN, a neutron imaging apparatus in J-PARC MLF under proposal number 2019B0229. The authors are grateful to Dr. Takenao Shinohara and Dr. Tetsuya Kai of JAEA for their constructive suggestions and support during the experiment.

\section{References}

[1] The Institute of Applied Energy, "Report on 2009 Energy Saving Equipment Introduction Promotion Guidance Project (Survey Business on the Actual Condition of Energy Consuming Equipment)," IAE-0919107, 2010 (in Japanese).

[2] Nitta, M., Tsuda, T., Arai, H., Sakamoto, K. and Sakai, K., "Effects of Transition Point of Viscoelasticity of Diurea Grease and Molecular Structure of Thickener on Running Torque of the Ball Bearing -Effects of Alkyl Chain Length of Aliphatic Diurea-," Journal of Japanese Society of Tribologists, 61, 10, 2016, $699-708$ (in Japanese).

[3] Cousseau, T., Graca, B., Campos, A. and Seabra, J., "Friction Torque in Grease Lubricated Thrust Ball Bearings," Tribology International, $44,5,2011,523-531$.

[4] Hutton, J. F., "The Influence of Flow Elasticity on the Bearing Performance of Lubricating Grease," Proc JSLE-ASLE International
Lubrication Conference, 1975, 707-714.

[5] Lugt, P. M., "Modern Advancements in Lubricating Grease Technology," Tribology International, 97, 2016, 467-477.

[6] Noda, T., Shibasaki, K., Miyata, S. and Taniguchi, M., "X-Ray CT Imaging of Grease Behavior in Ball Bearing and Numerical Validation of Multi-Phase Flows Simulation," Tribology Online, 15, 1, 2020, 36-44.

[7] Haruyama, T. and Sawada, N., "Visualization of Grease Flow Using Fluorescent Particle -2nd: Effect of Grease Characteristics-," Proc. JAST Tribology Conference, Tokyo, May 2017, E34, 361-364 (in Japanese).

[8] Ito, A. and Nose, H., "Visualization of Grease 3D Distribution in Rolling Bearing by Neutron Radiography," Proc. JAST Tribology Conference, Fukuoka, October 2013, B19 (in Japanese).

[9] Shinohara, T., Kai, T., Oikawa, K., Nakatani, T., Segawa, M., Hiroi, K., Su, Y., Ooi, M., Harada, M., Iikura, H., Hayashida, H., Parker, J. D., Matsumoto, Y., Kamiyama, T., Sato, H. and Kiyanagi, Y., “The Energy-Resolved Neutron Imaging System, RADEN," Review of Scientific Instruments, 91, 4, 2020, 043302.

[10] Nakajima, K., Kawakita, Y., Itoh, S., Abe, J., Aizawa, K., Aoki, H., Endo, H., Fujita, M., Funakoshi, K., Gong, W., Harada, M., Harjo, S., Hattori, T., Hino, M., Honda, T., Hoshikawa, A., Ikeda, K., Ino, T., Ishigaki, T., Ishikawa, Y., Iwase, H., Kai, T., Kajimoto, R., Kamiyama, T., Kaneko, N., Kawana, D., Ohira-Kawamura, S., Kawasaki, T., Kimura, A., Kiyanagi, R., Kojima, K., Kusaka, K., Lee, S., Machida, S., Masuda, T., Mishima, K., Mitamura, K., Nakamura, M., Nakamura, S., Nakao, A., Oda, T., Ohhara, T., Ohishi, K., Ohshita, H., Oikawa, K., Otomo, T., Sano-Furukawa, A., Shibata, K., Shinohara, T., Soyama, K., Suzuki, J., Suzuya, K., Takahara, A., Takata, S., Takeda, M., Toh, Y., Torii, S., Torikai, N., Yamada, N. L., Yamada, T., Yamazaki, D., Yokoo, T., Yonemura, M. and Yoshizawa, H., "Materials and Life Science Experimental Facility (MLF) at the Japan Proton Accelerator Research Complex II: Neutron Scattering Instruments," Quantum Beam Science, 1, 3, 2017, 9.

This paper is licensed under the Creative Commons Attribution-NonCommercial-NoDerivatives 4.0 International (CC BY-NC-ND 4.0) International License. This allows users to copy and distribute the paper, only upon conditions that (i) users do not copy or distribute such paper for commercial purposes, (ii) users do not change, modify or edit such paper in any way, (iii) users give appropriate credit (with a link to the formal publication through the relevant DOI (Digital Object Identifier)) and provide a link to this license, and (iv) users acknowledge and agree that users and their use of such paper are not connected with, or sponsored, endorsed, or granted official status by the Licensor (i.e. Japanese Society of Tribologists). To view this license, go to https://creativecommons.org/licenses/by-nc-nd/4.0/. Be noted that the third-party materials in this article are not included in the Creative Commons license, if indicated on the material's credit line. The users must obtain the permission of the copyright holder and use the third-party materials in accordance with the rule specified by the copyright holder. 